\title{
论文
}

\section{基于首次超越概率理论的混凝土坝时变风险率 研究}

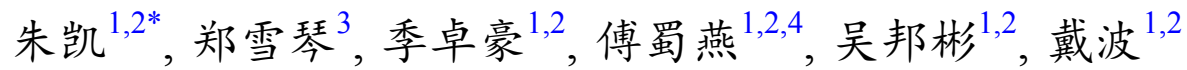

1. 河海大学水文水资源与水利工程科学国家重点实验室, 南京 210098;

2. 河海大学水资源高效利用与工程安全国家工程研究中心, 南京 210098;

3. 国网新源控股有限公司技术中心, 北京 100761 ;

4. 云南农业大学水利学院, 昆明 650201

*E-mail: hhukaiz@163.com

收稿日期: 2018-03-07; 接受日期: 2018-06-25; 网络版发表日期: 2018-12-06

国家重点研发计划(编号：2017YFC0804607)、国家自然科学基金重点项目(编号：51739003)、国家自然科学基金(批准号：51479054, 51779086, 51579086, 51379068, 51579083, 51579085, 51609074)、国家重点实验室专项基金(编号: 20165042112)、广西重点研发计划(编号: 桂科AB17195074)、江苏高校优势学科建设工程资助项目(水利工程)(编号：YS11001)、江苏省2015年度普通高校研究生科研创新计划(编号： KYZZ15_0140)、水文水资源与水利工程科学国家重点实验室开放基金(编号：2016491811，2017491811)和云南省教育厅科学研究基金(编号: 2016ZZX109)资助项目

摘要随着我国大坝安全管理工作逐渐由传统的工程安全管理向工程风险管理方向发展, 在役大坝风险水平成 为了越来越重要的研究课题. 混凝土坝实际运行过程中受到众多随机不确定因素和模糊因素影响, 导致大坝自身 风险率具有显著的时变特性, 本文尝试引入二元高斯算法采用并联体系方法计算混凝土坝系统时变风险率, 将时 变风险率分析问题转化为静态并联体系失效概率计算问题，通过在广义随机空间内采用 JC法计算固定时刻混凝 土坝风险率，并基于首次超越概率理论转化为混凝土坝时变风险率。最后，在工程算例分析中以某混凝土重力坝 沿坝基抗滑失效为例, 通过引入合理的抗力和荷载效应随机过程变量模型, 采用该算法对混凝土重力坝抗滑稳定 时变风险率进行了分析, 验证了该模型的有效性, 具有一定的理论和工程应用价值.

关键词混凝土坝, 时变风险率, 首次超越概率, 二元高斯方法, 随机过程模型

\section{1 引言}

混凝土坝通常建造于偏远的高山峡谷中，服役环 境恶劣，由于坝体直接与水、大气和基岩接触，大坝 混凝土在长期承受各种静、动荷载的同时，还需要长 期遭受高压渗流、溶蚀、冲刷、冻融和热胀冷缩等有

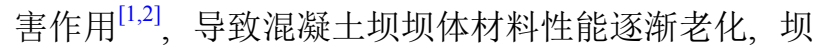
体整体服役性能劣化，大坝完成预定功能的能力下降， 大坝风险率不断增加 ${ }^{[3,4]}$. 国内外已开展大量关于混凝 土坝风险率分析研究工作，例如Vrijling等人 ${ }^{[5]}$ 采用直 接积分法对大坝漫顶以及溢流风险率进行了研究; Griffiths等人 ${ }^{[6]}$ 基于随机有限元模型，将弹塑性理论与

引用格式: 朱凯, 郑雪琴, 季卓豪, 等. 基于首次超越概率理论的混凝土坝时变风险率研究. 中国科学: 技术科学, 2019, 49: 331-342

Zhu K, Zheng X Q, Ji Z H, et al. The research of the time-varying risk of concrete dam based on first transcendental probability theory (in Chinese) Sci Sin Tech, 2019, 49: 331-342, doi: 10.1360/N092018-00044 
随机场相结合, 分析了混凝土坝边坡失稳风险率; Kuo 等人 ${ }^{[7]}$ 综合利用点估计法理论、蒙特卡罗法、拉丁超 立方抽样法以及一次二阶距法对Feitsui大坝进行了漫 坝风险率研究; 解家毕和孙东亚 ${ }^{[8]}$ 考虑各种影响因素 的不确定性, 基于事件树法对堤防可能出现的破坏模 式进行了风险分析; 雷瑞丽等人 ${ }^{[9]}$ 考虑水位变化条件 等不确定性因素对大坝风险的影响, 利用随机微分方 程量化分析了大坝漫顶风险率; 彭慧慧 ${ }^{[10]}$ 针对重力坝 抗滑稳定性, 研究了各影响因素随机性和模糊性, 进 行了大坝失稳模糊风险率研究. $\mathrm{Hu}$ 等人 ${ }^{[11]}$ 通过采用矩 阵融合方法考虑不同失效模式之间的相关性, 提出了 基于非线性有限元理论的混凝土坝系统失效概率计算 方法, 并基于开源可靠度分析软件FERUM和开源有限 元软件Code_Aster(CA)构建了混凝土坝系统失效概率 分析计算平台 ${ }^{[11]}$. 相较上述大坝静态风险率研究, 我国 大坝时变风险率课题研究起步较晚, 2005年, 徐卫军等 人 $^{[12]}$ 基于灰色理论, 将结构时变可靠度预测和灰色关 联度相结合应用于结构可靠度分析中, 通过扩展荷载 时间序列定量计算结构时变可靠度指标与相关荷载的 关联程度; 姜树海和范子武 ${ }^{[13]}$ 在大坝工程老化的研究 中考虑了时变效应, 评估了影响防洪安全的各种不确 定性因素时变特性，建立了防洪时变风险率模型; 苏 怀智等人 ${ }^{[14]}$ 基于模糊事件概率理论, 针对大坝运行过 程中各种风险因子的时变特性, 结合信息熵理论, 建 立了大坝模糊时变风险率分析模型. 总体来讲, 关于 大坝时变风险率研究相对较少, 还没有形成系统理论 体系. 事实上, 在混凝土坝实际运行过程中, 由于大坝 荷载和抗力受各种不确定因素和随机因素的作用, 导 致大坝自身材料参数及服役荷载条件均随着时间或空 间而变化, 相应大坝风险率也动态调整, 本文尝试引入 时变风险率理论对在役混凝土坝服役过程时变失效概 率进行分析, 基于首次超越概率原理, 利用二元高斯算 法计算混凝土坝失效正斜率跨越率, 并结合混凝土坝 自身抗力模型和荷载效应失效统计结果, 进行混凝土 坝时变风险率研究.

\section{2 基本理论}

\section{1 大坝时变风险率基本理论}

混凝土坝时变风险率分析模型可以通过将结构的 极限状态方程赋予时间属性获得, 相应混凝土坝动态
服役性能函数可以表示如下:

$$
Z(t)=R(t)-S(t)=F\left(X_{1}, X_{1}, \ldots, X_{n}, t\right),
$$

式中, $R(t)$ 和 $S(t)$ 分别为结构抗力随机变量和荷载效应 随机变量; $X_{1}, X_{2}, \ldots, X_{n}$ 为结构的几何尺寸、材料的物 理参数以及结构所受的荷载作用等时变随机变量. 若 假设上述随机变量 $X_{1}, X_{2}, \ldots, X_{n}$ 的联合概率密度函数为 $f\left(x_{1}, x_{2}, \ldots, x_{n}, t\right)$, 则相应混凝土坝结构时变风险率可以 计算为

$$
\begin{aligned}
p_{f}(t) & =p(Z(t)<0) \\
& =\iint_{Z<0} \cdots \int f_{X}\left(x_{1}, x_{2}, \ldots, x_{n}\right) \mathrm{d} x_{1} \mathrm{~d} x_{2} \cdots \mathrm{d} x_{n} .
\end{aligned}
$$

水工混凝土结构荷载按照作用效应可以分为永久 荷载和可变荷载, 若假设混凝土坝永久荷载为 $S_{G}$, 可变 荷载为 $S_{Q}(t)$, 则相应极限状态函数可以表示如下:

$Z(t)=R(t)-S_{G}-S_{Q}(t)$.

在设计服役基准期内，相应混凝土坝结构风险率 可以表示如下:

$$
\begin{aligned}
p_{f}\left(t_{s}\right) & =P\left\{Z(t)=R(t)-S_{G}-S_{Q}(t)<0, t \in\left[0, t_{s}\right]\right\} \\
& =P\left\{\min \left[R(t)-S_{G}-S_{Q}(t)<0\right], t \in\left[0, t_{s}\right]\right\} .
\end{aligned}
$$

如式(4)所示, 混凝土坝结构时变风险率涉及众多 随机变量, 计算时涉及高维积分问题, 难以直接应用. 基于以上问题, 德国慕尼黑理工大学的 Schrupp 和 Rackwitz提出了首次超越概率方法, 该方法将结构功 能函数视为一组动力随机过程, 并基于首次超越破坏 准则 (当结构反映在给定的时间段内首次超越安全界 限即认为结构失效), 通过假定结构随机反应与安全界 限的交差符合某种特殊分布, 将包含随机过程的极限 状态方程失效概率计算转化为跨越率计算问题, 进而 推求结构时变风险率.

基于首次超越概率理论, 功能函数 $F\left(X_{1}, X_{2}, \ldots, X_{n}\right)$ 跨越安全界限的次数服从Poisson分布, 每次跨越过程 相互独立. 设 $X(t)$ 为一连续状态随机过程, 构造0-1过 程 $Y(t)$ 如下:

$$
Y(t)=U[X(t)-b],
$$

式中, $U[X(t)-b]$ 为单元阶跃函数; $b$ 为界限值. 相应在 $(0, T)$ 时间范围内随机变量 $X(t)$ 跨越界限值 $b$ 的次数可 以表示如下: 


$$
\begin{aligned}
N_{b}(0, T) & =\int_{0}^{T} E|\dot{X}(t)| \delta[X(t)-b] \mathrm{d} t \\
& =\int_{0}^{T} \int_{-\infty}^{+\infty} \int_{-\infty}^{+\infty}|\dot{x}| \delta(x-b) p(x, \dot{x}, t) \mathrm{d} x \mathrm{~d} \dot{x} \mathrm{~d} t,
\end{aligned}
$$

式中, $\delta(\cdot)$ 为狄拉克函数; $X(t), \dot{X}(t)$ 分别表征 2 组随机过 程. 假定正、负斜率跨越界限的次数相等, 相应正斜率 跨越率可以表示为

$$
v^{+}(t)=\int_{-\infty}^{+\infty} x p(b, \dot{x}, t) \mathrm{d} \dot{x} .
$$

在首次超越破坏准则下, 随机过程失效概率即为 在给定时间 $(0, T)$ 内首次超越安全界限的概率, 基于 Poisson分布假设, 当 $n=0$ 时, 随机变量 $X(t)$ 在时间段 $[t, \Delta t]$ 从不跨越界限的概率为 ${ }^{[15]}$

$$
\begin{aligned}
P(0, t) & =\exp \left[\int_{0}^{t} v^{+}(t) \mathrm{d} t\right] \\
& =C_{n}+\int_{0}^{t} P(-1) v^{+}(t) \exp \left[\int_{0}^{t} v^{+}(u) \mathrm{d} u\right] \mathrm{d} t .
\end{aligned}
$$

假定随机变量在初始状态不发生跨越, 即 $t=0$ 时, $P(0,0)=1$, 因此可以推求 $C_{0}=1$, 相应混凝土坝时变失 效概率可以计算如下:

$p_{f}(t)=1-\exp \left[-\int_{0}^{t} v^{+}(t) \mathrm{d} t\right] \mathrm{d} t$.

因此，采用首次超越概率方法计算混凝土坝时变 风险率的关键在于求解混凝土坝失效事件正斜率跨越 率 $v^{+}(t)$, 进而基于式(9)在设计基准期或者剩余使用寿 命期内对期望跨越率进行积分，即可计算混凝土坝时 变失效概率.

\section{2 二元高斯方法基本原理}

Hagen和Tvedt ${ }^{[16 \text { - 18] }}$ 于1991年提出采用并联体系方 法计算时变系统跨越率，并在后期广泛应用于非线性 动力系统和非线性空间变形有限元分析. 基于上述跨 越率计算思路, Andrieu-Renaud等人 ${ }^{[19]}$ 在2004年提出 了二元高斯方法(PHI2 Method, 其中PHI为希腊语表 征标准正态分布，PHI_2表征二元高斯概率分布函数, 下文简称为PHI2), 该方法将跨越率的计算问题转化为 静态的并联体系的失效概率问题，可以高效求解任意
时变问题的跨越率计算问题.

假定混凝土坝极限状态方程为 $G(t, X(t))$, 当 $G(t, X$ $(t))>0$ 时, 代表结构位于安全区域, 当 $G(t, X(t))<0$ 时, 代 表结构位于危险区域, 混凝土坝在 $(0, t)$ 时间段内失效 事件可以表示如下:

$E=\{\exists \tau \in[0, t] \mid G(\tau, \omega) \leq 0\}$,

式中, $\omega$ 代表影响混凝土坝安全性态的随机变量集合. 相应混凝土坝累计失效概率可以表示如下:

$$
\begin{aligned}
p_{f, c}(0, t) & =\operatorname{Prob}(E) \\
& =\operatorname{Prob}(\exists \tau \in[0, t] \mid G(\tau, X(\tau, \omega) \leq 0) .
\end{aligned}
$$

当采用首次超越概率方法计算混凝土坝系统失效 概率时, 式(11)可以进一步修改为 ${ }^{[20]}$

$$
\begin{aligned}
p_{f}\left(t_{0}, t_{s}\right)= & \operatorname{Pr}\left\{Q\left(t_{0}\right)>0\right\} \\
& +\operatorname{Pr}\left\{Q\left(t_{0}\right)<0\right\} \\
& \times \operatorname{Pr}\left\{Q(\tau)>0, \exists \tau \in\left[t_{0}, t_{s}\right]\right\},
\end{aligned}
$$

或

$$
\begin{aligned}
p_{f}\left(t_{0}, t_{s}\right)= & \operatorname{Pr}\left\{Q\left(t_{0}\right)>0\right\} \\
& +\operatorname{Pr}\left\{Q\left(t_{0}\right)<0\right\} \int_{t_{0}}^{t_{s}} f_{T_{1}}(t) \mathrm{d} t,
\end{aligned}
$$

式中, $f_{T_{1}}(t)$ 为首次失效(first time to failure, FTTF)事件 概率密度函数.

由上式可知, 混凝土坝失效包括两种可能, 一种可 能为混凝土坝在 $t=0$ 时刻, 即初始时刻 $t_{0}$ 发生失效; 另一 种可能为混凝土坝初始时刻不失效, 仅在 $t>0$ 时段内发 生失效，相应混凝土坝失效概率上下界限可以表示 如下:

$$
\max _{0 \leq \tau \leq t}\left(p_{f, i}(\tau)\right) \leq p_{f, c}(0, t) \leq p_{f, i}(0)+E\left[N^{+}(0, t)\right] .
$$

基于以上分析, 相应定义跨越率 $v^{+}(t)$ 变量如下:

$v^{+}(\tau)=\lim _{\Delta \tau \rightarrow 0, \Delta \tau>0} \frac{P\left[N^{+}(\tau, \tau+\Delta \tau)=1\right]}{\Delta \tau}$,

式中 $P\left[N^{+}(\tau, \tau+\Delta \tau)=1\right]$ 表征极限状态方程在 $[\tau, \tau+\Delta \tau]$ 时 段内发生一次跨越的概率, 基于条件概率理论, 上式可 以进一步修改为

$v^{+}(\tau)=\lim _{\Delta \tau \rightarrow 0, \Delta \tau>0} \frac{\operatorname{Prob}(\{G(\tau, X(\tau, \omega))>0\} \cap\{G(\tau+\Delta \tau, X(\tau+\Delta \tau, \omega)) \leq 0\})}{\Delta \tau}$. 
式中函数极限 $\Delta \tau \rightarrow 0$ 表示在时间段 $[\tau, \tau+\Delta \tau]$ 内极限状 中的函数极限形式, PHI2定义的跨越率表达式如下:

态方程发生超过一次跨越的概率为 0 , 通过去除式(16)

$\nu_{\mathrm{PHI} 2}^{+}(\tau)=\frac{\operatorname{Prob}(\{G(\tau, X(\tau, \omega))>0\} \cap\{G(\tau+\Delta \tau, X(\tau+\Delta \tau, \omega)) \leq 0\})}{\Delta \tau}$.

因此, 通过合理选择 $\Delta \tau$, PHI2方法可以将时变风 险率计算问题转化为两个固定时刻的时不变可靠度计 算问题，通过式(17)即可将跨越率的计算转换为静态 并联体系的失效概率计算问题，避免了采用式(7)积分 方法计算混凝土坝极限状态方程跨越率，将有效提高 算法求解效率和精度. 本文拟采用广义随机空间内 JC 法计算各时间点静态可靠度, 相应对该算法说明如下: $\mathrm{JC}$ 法的理论基础为一次二阶矩法，假定结构原始功能 函数为 $Z=G\left(x_{1}, x_{2}, \ldots, x_{n}\right)$, 通过对各随机变量进行标准 正态化 $U_{i}=\frac{X_{i}-\mu_{X_{i}}}{\sigma_{X_{i}}}$, 并不会改变各随机变量之间的相 关性, 相应极限状态方程可以修改为 $Z=f_{1}\left(U_{1}, U_{2}, \ldots, U_{n}\right)$, 事实上, 在随机变量 $U$ 空间中, 功能函数 $Z=G\left(x_{1}, x_{2}, \ldots\right.$, $\left.x_{n}\right)$ 为非线性超曲面，可靠度指标的即为从原点 $M\left(O_{1}\right.$, $\left.O_{2}, \ldots, O_{n}\right)$ 到极限状态曲面 $Z=0$ 的最短距离, 相应离原 点最近的点称为设计验算点 $P^{*}\left(U_{1}^{*}, U_{2}^{*}, \ldots, U_{n}^{*}\right)$, 通 过 $P^{*}$ 点的超曲面切平面方程可以表示如下:

$$
Z^{\prime}=f_{1}\left(U_{1}^{*}, U_{2}^{*}, \ldots, U_{n}^{*}\right)+\left.\sum_{i=1}^{n} \frac{\partial f_{1}}{\partial U_{i}}\right|_{U_{i}^{*}} \cdot\left(U_{i}-U_{i}^{*}\right) .
$$

可靠度指标 $\beta$ 为从原点 $\mathrm{M}$ 到超曲面的最短距离, 可 以表示如下:

$$
\beta=\frac{-\left.\sum_{i=1}^{n} \frac{\partial f_{1}}{\partial U_{i}}\right|_{U_{i}^{*}} \cdot U_{i}^{*}}{\sqrt{\sum_{i=1}^{n}\left(\left.\frac{\partial f_{1}}{\partial U_{i}}\right|_{U_{i}^{*}}\right)^{2}}} .
$$

在解决含有相关随机变量的结构可靠度问题时, 常规方法往往采用正交变换方法把相关随机变量变换 为不相关随机变量, 计算较为繁琐, 本文尝试在广义随 机空间内基于等概率变换原则在广义随机空间对各相 关随机变量进行转换 ${ }^{[21,22]}$, 对混凝土坝极限状态方程 内部各随机变量进行当量正态化处理后, 将功能函数 $Z=G\left(x_{1}, x_{2}, \ldots, x_{n}\right)$ 在设计验算点 $x^{*}=\left(x_{1}^{*}, x_{2}^{*}, \ldots, x_{n}^{*}\right)$ 采 用Taylor级数展开可得

$$
Z=G\left(x_{1}^{*}, x_{2}^{*}, \ldots, x_{n}^{*}\right)+\left.\sum_{i=1}^{n} \frac{\partial G}{\partial X_{i}}\right|_{x^{*}}\left(X_{i}^{\prime}-x_{i}^{*}\right),
$$

式中, $\left.\frac{\partial G}{\partial X_{i}}\right|_{x^{*}}$ 为功能函数导数在验算点处的值.

由于对非正态随机变量进行当量正态化处理并不 改变随机变量间的线性相关性, 即 $\rho_{X_{i}^{\prime} X_{j}^{\prime}} \approx \rho_{X_{i} X_{j}}$, 由正 态分布随机变量特性可得当量正态化处理后功能函数 的均值和标准差为

$$
\begin{aligned}
\mu_{Z^{\prime}} & =\left.\sum_{i=1}^{n} \frac{\partial G}{\partial X_{i}}\right|_{x^{*}}\left(\mu_{X_{i}^{*}}-x_{i}^{*}\right), \\
\sigma_{Z} & =\left(\left.\sum_{i=1}^{n} \sum_{j=1}^{n} \frac{\partial G}{\partial X_{i}} \frac{\partial G}{\partial X_{j}}\right|_{x^{*}} \rho_{X_{i} X_{j}} \sigma_{X_{i}} \sigma_{X_{j}^{\prime}}\right)^{1 / 2} \\
& =-\left.\sum_{i=1}^{n} \alpha_{i}^{\prime} \frac{\partial G}{\partial X_{i}}\right|_{x^{*}} \sigma_{X_{i}^{\prime}},
\end{aligned}
$$

式中, $\alpha_{i}^{\prime}$ 为灵敏系数, 反映了随机变量 $X_{i}{ }_{i}$ 对功能函数 $Z$ 的影响程度, 其表达式如下:

$$
\alpha_{i}^{\prime}=\frac{\left.\sum_{j=1}^{n} \frac{\partial G}{\partial X_{j}}\right|_{x^{*}} \rho_{X_{i} X_{j}} \sigma_{X_{j}^{\prime}}}{\left(\left.\sum_{j=1}^{n} \sum_{k=1}^{n} \frac{\partial G}{\partial X_{j}} \frac{\partial G}{\partial X_{k}}\right|_{x^{*}} \rho_{X_{j} X_{k}} \sigma_{X_{j}^{\prime}} \sigma_{X_{k}^{\prime}}\right)^{1 / 2} .}
$$

将式(21) (23)代入式(20), 即可求出验算点公式 如下:

$$
x_{i}^{*}=\mu_{X_{i}^{\prime}}+\beta \alpha_{i}^{\prime} \sigma_{X_{i}^{\prime}} \text {. }
$$

因此, 通过在广义随机空间内联立式(23)和(24)进 行迭代计算, 即可求出结构在各固定时间点对应的静 态可靠度及其对应的风险率.

\section{3 基于二元高斯方法的混凝土坝时变风险率分 析步骤}

由上述分析可知，本文通过引入二元高斯方法将 时变风险率分析问题转化为静态并联体系失效概率计 
算问题, 通过固定时间 $\tau$, 并合理选择 $\Delta \tau$, 在广义随机空 间内采用JC法计算两个固定时刻混凝土坝风险率，并 基于首次超越概率理论结合式(17)计算跨越率 $v^{+}(t)$, 最 终基于式(9)即可对混凝土坝时变风险率进行计算，基 本计算流程如图1, 可以总结如下.

(1) 通过固定时间 $t=\tau$, 通常取为 $t=0$, 相应时变随机 变量 $X_{k}(t, \omega)$ 可以转化为时不变随机变量 $X_{k}^{1}(\omega)$, 并基 于混凝土坝极限状态方程 $\{G(\tau, X(\tau, \omega)) \leq 0\}$ 在广义随 机空间内采用 JC法计算结构可靠度 $\beta(\tau)$.

(2) 通过合理选择时间间隔 $\Delta \tau$, 可以将时变随机变 量 $X_{k}(t, \omega)$ 转化为时不变随机变量 $X_{k}^{2}(\omega)$, 可以采用广 义随机空间内部定义的 $\mathrm{JC}$ 法计算对应于混凝土坝极 限状态方程 $\{G(\tau+\Delta \tau, X(\tau+\Delta \tau, \omega)) \leq 0\}$ 的结构可靠度 $\beta(\tau+\Delta \tau)$, 相应两组时不变随机变量相关系数可以表 示为

$$
\rho\left(X_{k}^{(1)}, X_{k}^{(2)}\right)=\rho_{X_{k}}(\tau, \tau+\Delta \tau) .
$$

假设时变随机变量 $X_{k}(t, \omega)$ 服从标准正态分布, 任 意两组时不变随机变量自相关系数为 $\rho_{X_{k}}\left(t_{1}, t_{2}\right)$, 则可
以采用Cholesky分解将两组随机变量 $\left\{X_{k}^{(1)}, X_{k}^{(2)}\right\}$ 转化 为相互独立随机变量, 相应Cholesky矩阵为

$\left(\begin{array}{cc}1 & \rho_{X_{k}}(\tau, \tau+\Delta \tau) \\ \rho_{X_{k}}(\tau, \tau+\Delta \tau) & 1\end{array}\right)$.

相应随机变量转化过程如下:

$\left\{\begin{array}{l}X_{k}^{(1)}=\xi_{1}, \\ X_{k}^{2}=\rho_{X_{k}}(\tau, \tau+\Delta \tau) \xi_{1}+\sqrt{1-\rho_{X_{k}}^{2}(\tau, \tau+\Delta \tau)} \xi_{2},\end{array}\right.$

式中, $\xi_{1}$ 和 $\xi_{2}$ 为相互独立正态随机变量.

(3) 求解任意两个时刻大坝失效极限状态方 程 $\{G(\tau, X(\tau, \omega)) \leq 0\}$ 与 $\{G(\tau+\Delta \tau, X(\tau+\Delta \tau, \omega)) \leq 0\}$ 相 关系数 $\rho_{G}(\tau, \tau+\Delta \tau)=-\alpha(\tau) \cdot \alpha(\tau+\Delta \tau)$, 式中 $\alpha(\cdot)$ 表示两 组时刻极限状态方程对应超曲面在设计验算点处的单 位法向量.

传统JC方法无法计算不同时刻极限状态方程相 关系数 $\rho_{G}(\tau, \tau+\Delta \tau)$, 本文采用Pearson相关系数方程 ${ }^{[19]}$ 进行求解. 假设存在两组 $n$ 维样本集合 $A=\left\{A_{i}\right\}$ 和 $B=$ $\left\{B_{i}\right\}$, 其中样本集合 $A$ 表征极限状态方程 $G(\tau, X(\tau, \omega))$,

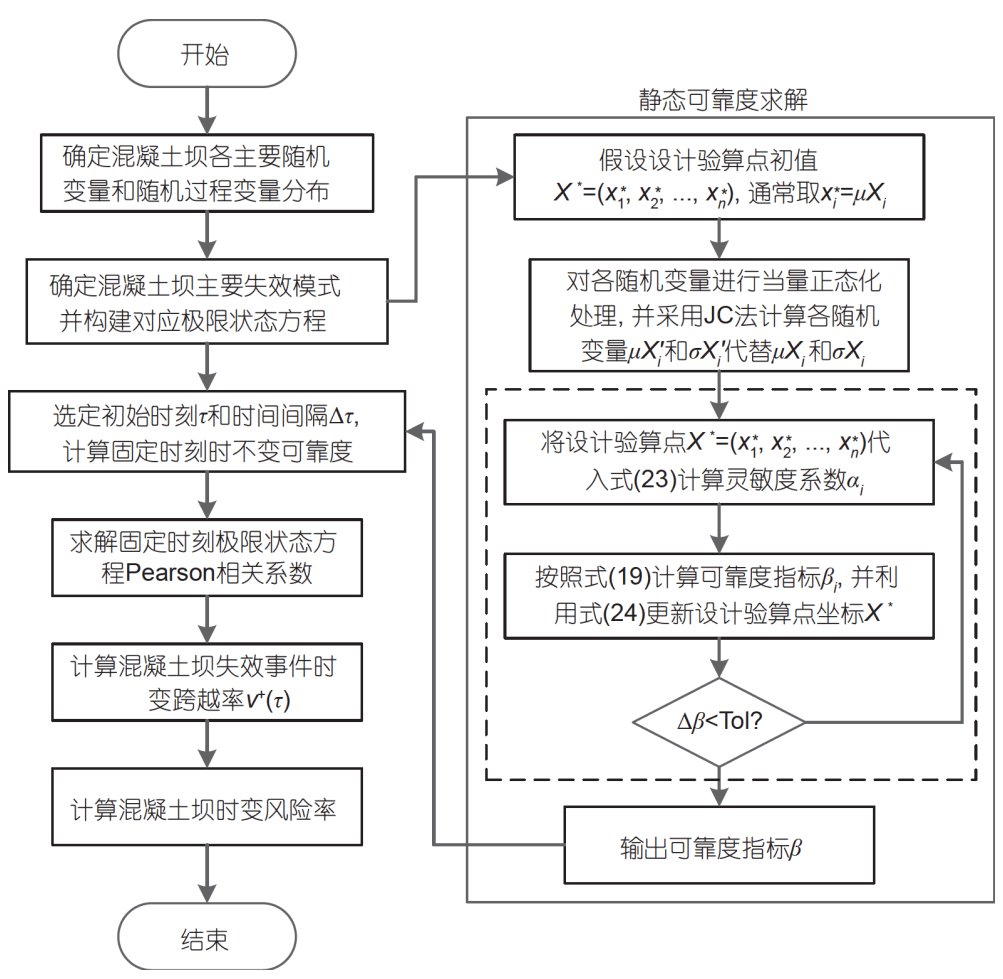

图 1 基于PHI2方法的混凝土坝时变风险率求解示意图 
样本集合 $B$ 表征极限状态方程 $G(\tau+\Delta \tau, X(\tau+\Delta \tau, \omega))$, 则 不同时刻极限状态方程相关系数 $\rho_{G}(\tau, \tau+\Delta \tau)$ 可以表示 如下:

$$
\rho_{G}(\tau, \tau+\Delta \tau)=\frac{\operatorname{Cõv}(A, B)}{\sqrt{\operatorname{Vãr}(A)} \sqrt{\operatorname{Vãr}(B)}},
$$

式中, 样本集合 $A$ 和 $B$ 协方差变量

$\operatorname{Cõv}(A, B)=\frac{1}{n-1} \sum_{i=0}^{n-1}\left(A_{i}-\widetilde{m}_{A}\right)\left(B_{i}-\widetilde{m}_{B}\right) ;$
标准差

$$
\begin{aligned}
& \operatorname{Vãr}(A)=\frac{1}{n-1} \sum_{i=0}^{n-1}\left(A_{i}-\widetilde{m}_{A}\right)^{2}, \\
& \operatorname{Vãr}(B)=\frac{1}{n-1} \sum_{i=0}^{n-1}\left(B_{i}-\widetilde{m}_{B}\right)^{2} ; \\
& \text { 均值 } \widetilde{m}_{A}=\frac{1}{n} \sum_{i=0}^{n-1} A_{i}, \widetilde{m}_{B}=\frac{1}{n} \sum_{i=0}^{n-1} B_{i} .
\end{aligned}
$$

综上, 混凝土坝时变跨越率可以表示如下:

$v^{+}(\tau)=\frac{P(\{G(X(\tau), \tau)>0\} \cap\{G(X(\tau+\Delta \tau), \tau+\Delta \tau>0)\} \leq 0)}{\Delta \tau}=\frac{\Phi\left[\beta(\tau),-\beta(\tau+\Delta \tau) ; \rho_{G}(\tau, \tau+\Delta \tau)\right]}{\Delta \tau}$,

式中, $\beta(\tau)$ 和 $\beta(\tau, \tau+\Delta \tau)$ 为混凝土坝任意两个时刻的可靠 度指标; $\Phi(\cdot)$ 为二维标准正态分布函数.

(4) 基于式(9)计算混凝土坝时变风险率.

\section{3 在役混凝土坝水位荷载效应统计特性 分析}

水压力是水工建筑物的主要可变荷载, 对应荷载 模型选取合理与否, 将直接影响大坝风险预测及可靠 性评估准确性. 通常情况下，多年运行混凝土坝具有 十分详细的水库水位观测资料，因此可以基于大坝监 测资料分析，确定大坝年最高水位分布函数 $F_{h}(x)$. 基 于文献[23]，通过假设服役混凝土坝继续服役基准期 为 $T^{\prime}$, 可以采用年最高水位的分布函数 $F_{h}(x)$ 推求继续 服役基准期 $T^{\prime}$ 内极值最高水位 $H_{\mathrm{max}}$ 的概率密度函 数 $f_{T^{\prime}}(x)$ 和分布函数 $F_{T^{\prime}}(x)$. 首先假定混凝土坝水库各 年最高水位相互独立，基于“等超越概率”原则，继续 服役基准期 $T^{\prime}$ 内极值最高水位的分布函数可以表示 如下:

$$
F_{T^{\prime}}(x)=\left[F_{h}(x)\right]^{\frac{N T}{M}},
$$

式中, $T$ 为混凝土坝设计服役基准期.

通常情况下，库水位分量在混凝土坝极限状态方 程中显示存在，直接采用式(30)计算 $F_{T^{\prime}}(x)$ 的统计参数 过程较为复杂, 基于已有研究资料分析, 对于库水位 呈年周期性分布的水库工程, 可以认为 $F_{T^{\prime}}(x)$ 和 $F_{h}(x)$ 均以正态分布拟合较好，基于此，上式可以进一步表 示为
$\Phi\left(\frac{x-\mu_{T^{\prime}}}{\sigma_{T^{\prime}}}\right)=\left[\Phi\left(\frac{x-\mu_{h}}{\sigma_{h}}\right)\right]^{m_{T^{\prime}}}$,

式中, $m_{T^{\prime}}$ 为在继续使用期 $M$ 年内出现年最高水位的次 数, 此时 $T^{\prime}=m_{T^{\prime}}$, 即每年出现一次, 出现次数与时段数 相同, 且 $\tau=1$ 年.

基于最小二乘方法，假定存在两组固定点 $V_{1}$ 和 $V_{2}$, 使得

$\left\{\begin{array}{l}x_{1}=\mu_{T^{\prime}}+V_{1} \sigma_{T^{\prime}}, \\ x_{2}=\mu_{T^{\prime}}+V_{2} \sigma_{T^{\prime}} .\end{array}\right.$

将式(32)代入式(31)可得

$\left\{\begin{array}{l}\mu_{T^{\prime}}+V_{1} \sigma_{T^{\prime}}=\mu_{h}+\Phi^{-1}\left[m_{T^{\prime}} \sqrt{\Phi\left(V_{1}\right)}\right] \sigma_{h}, \\ \mu_{T^{\prime}}+V_{2} \sigma_{T^{\prime}}=\mu_{h}+\Phi^{-1}\left[m_{T^{\prime}} \sqrt{\Phi\left(V_{2}\right)}\right] \sigma_{h^{\prime}} .\end{array}\right.$

联立上式可以求得

$\left\{\begin{array}{l}\mu_{T^{\prime}}=\mu_{h}+\frac{V_{2} \Phi^{-1}\left[m_{T^{\prime}} \sqrt{\Phi\left(V_{1}\right)}\right]-V_{1} \Phi^{-1}\left[m_{T^{\prime}} \sqrt{\Phi\left(V_{2}\right)}\right]}{V_{2}-V_{1}} \sigma_{h}, \\ \sigma_{T^{\prime}}=\frac{\Phi^{-1}\left[m_{T^{\prime}} \sqrt{\Phi\left(V_{2}\right)}\right]-\Phi^{-1}\left[m_{T^{\prime}} \sqrt{\Phi\left(V_{1}\right)}\right]}{V_{2}-V_{1}} \sigma_{h},\end{array}\right.$

式中, $\Phi(\cdot)$ 和 $\Phi^{-1}(\cdot)$ 分别为标准正态分布函数及其反函 数; $\mu_{h}$ 和 $\sigma_{h}$ 分别为 $F_{h}(x)$ 的均值和标准差.

因此，可通过实测的年最高水位的分布和统计参 数推求随机变量库水位 $H$ 的统计参数, 并直接应用 $\mathrm{JC}$ 法参与混凝土坝时变风险率计算 ${ }^{[23]}$. 通常情况下, 混 凝土坝具有详细的水位观测资料, 可直接根据实测年 最高水位统计分析在相应设防标准下(即继续服役基 
准期内)最高洪水位推求库水位 $H$ 分布特征.

\section{4 算例分析}

\section{1 工程简介}

本文以福建某混凝土重力坝 5 \#坝段沿建基面滑动 失稳为例, 说明PHI2方法在分析混凝土重力坝抗滑稳 定时变风险率中的应用. 该水库位于福建省金溪干流, 水库最大库容 8.54 亿立方米. 水库正常蓄水位 $275 \mathrm{~m}$, 校核洪水位为 $278.3 \mathrm{~m}$, 相应该水库多年库水位时程曲 线如图2, 由图可知, 该水库水位呈明显的年周期性变 化规律. 电站由拦河大坝、坝后溢流式厂房、埋设于 坝内的输水系统、泄水底孔及过木筏等组成. 大坝为 碾压混凝土重力坝，属于二级永久建筑物，坝顶高程 $280 \mathrm{~m}$, 最大坝高 $78 \mathrm{~m}$, 坝顶全长 $253 \mathrm{~m}$, 共分 13 个坝 段. 相应该坝典型坝段受力示意图如图 3 , 其中 $H_{1}$ 为上 游水深; $H_{2}$ 为下游水深; $H_{3}$ 为坝前泥沙淤积高程; $n$ 和 $m$ 分别为大坝上下游坡率; $W_{1}$ 为坝体自重; $W_{2}$ 和 $W_{3}$ 分别 上下游水深作用于坝体的重量; $P_{1}$ 和 $P_{2}$ 分别为大坝上 下游静水压力; $U$ 为扬压力.

\section{2 混凝土坝抗力和荷载效应随机过程变量拟定}

由于该混凝土坝尺寸巨大，假定坝身尺寸在服役 过程中保持不变, 即取为确定性变量. 对于其他影响 混凝土坝结构抗力和荷载效应随机过程变量, 本文选 定基本时变模型如下:

$X_{i}(t)=X_{i}(0) \varphi_{i}(t), \quad(i=1,2, \ldots, m)$,

式中, $\varphi_{i}(t)$ 为混凝土坝极限状态方程中第 $i$ 组变量演化 方程; $X_{i}(0)$ 和 $X_{i}(t)$ 分别为初始时刻和 $t$ 时刻随机变量; $t$ 为时间, 单位为a. 混凝土坝在外界侵蚀性环境以及荷 载作用下, 坝体材料会发生老化现象, 导致材料物理力 学性能, 如容重、抗拉强度、弹性模量、摩擦系数和 黏聚力等呈下降趋势，而相应的荷载因素如坝基扬压 力、泥沙容重和上游水位有可能随服役时间的增长而
增长, 因此, 需要结合工程实际制定合理的随机变量演 化方程 $\varphi_{i}(t)$.

混凝土坝坝基面力学材料参数(包括抗剪断摩擦 系数 $f^{\prime}$ 和黏聚力 $c^{\prime}$ ) 受众多复杂因素影响, 目前已有研究 成果中关于坝体混凝土/基岩胶结面间真实力学性质 的相关信息较少, Knodel等人 ${ }^{[24]}$ 采用实验方法测定了 混凝土坝基岩胶结面接触和强度参数, 并推求了在真 实应力状态下的大坝基岩胶结面强度破坏线; 美国电 力研究协会(Electric Power Research Institute, EPRI) ${ }^{[25]}$ 通过对建于1912 1974年间高为30 170 $\mathrm{m}$ 的17座不同 基岩类型胶结面钻孔样本实验研究，得到了大坝混凝 土/基岩胶结面的抗剪切和抗拉强度; 王宏硕等人 ${ }^{[26]}$ 基 于国内外野外抗剪断试验，将光弹实验方法和有限元 法相结合，对典型混凝土与基岩弹模比条件下建基面 应力进行了分析计算, 研究了试块与基岩胶结面上的 应力分布规律，提出了确定混凝土坝建基面真实抗剪 强度的方法和计算用表. 张俊芝和李桂青 ${ }^{[27]}$ 指出混凝 土坝-基岩胶结面性能劣化是一个复杂的物理、化学 和力学损伤过程, 除非外界环境非常严酷, 该过程极为 缓慢, 由于实测资料缺乏, 目前还无法对其演化过程进 行明确表述, 国内外普遍采用指数衰减函数描述上述 两组随机过程变量演化过程 ${ }^{[28,29]}$ ，本文结合文献[30] 假定两组随机过程变量具有相同的衰减规 律为 $\varphi_{1}(t)=\mathrm{e}^{-0.0005 t}$.

相同原理, 取该混凝土坝坝基扬压力折减系数 $\alpha$ 递 增函数为 $\varphi_{2}(t)=\frac{1}{2}+\frac{A_{1}}{1+B_{1} \mathrm{e}^{-0.005 t}}$, 式中 $A_{1}$ 和 $B_{1}$ 可以通过 历年坝基扬压力监测资料拟合求出. 相应该坝 5 \#坝段 坝基扬压力2011 2016年实测过程线如图4, 基于统计 检验理论, 本文选取两组参数分别为 $A_{1}=5.32, B_{1}=4.28$.

荷载效应随机过程变量本文主要考虑上游水深 $H_{1}$ 、下游水深 $H_{2}$. 其中, 1995 2014年上游历年年最高 水位统计结果如表 1 , 相应最高水位统计参数均值 $\mu_{h}=274.46$, 标准差 $\sigma_{h}=1.99$. 由于大坝下游水位相对变 化较为平稳, 因此, 本文将下游水位 $H_{2}$ 视为时不变随机

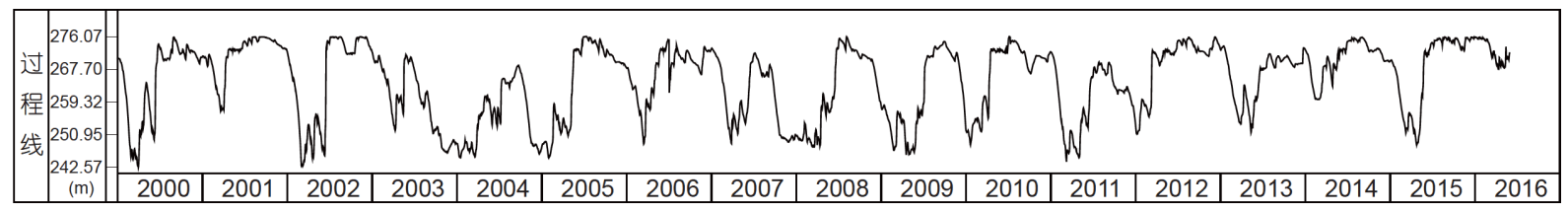

图 2 多年库水位时程曲线 


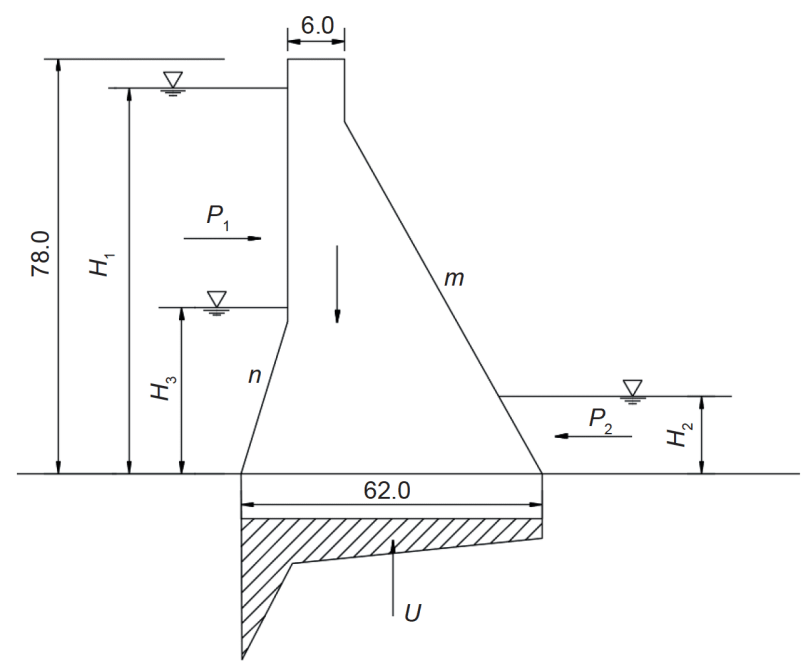

图 3 某混凝土重力坝非溢流坝段典型剖面图

变量, 相应统计特性如表2.

其他主要随机变量和统计参数见表 2 .

\section{3 基于PHI2 方法的混凝土重力坝时变风险率 计算}

综合上述随机变量定义, 并结合该混凝土坝实际 尺寸, 对于单位宽度坝体厚度, 可以得出混凝土重力 坝沿坝基面抗滑稳定的极限状态方程如下:

$$
\begin{aligned}
Z_{1}= & G\left(H_{1}, H_{2}, H_{3}, \gamma_{c}, f^{\prime}, c^{\prime}, U, n, m\right) \\
& =c^{\prime} A+f^{\prime}\left(\sum W-U\right)-\sum P \\
& =\left[2049.57 \gamma_{c}+4.87 H_{1}+0.4 H_{2}^{2}\right. \\
& \left.-317.5 \alpha\left(H_{2}-H_{1}\right)\right] f^{\prime}+62.0 c^{\prime}-5 H_{1}^{2}+5 H_{2}^{2} .
\end{aligned}
$$

本文选定式(25)中 $\Delta \tau=1$ 个月, 即 0.083 a. 首先基于 广义随机坐标空间, 采用 JC法计算不同时段的大坝抗 滑失稳可靠性指标, 如表 3 , 相应各时间段 JC推求的极 限状态方程验算点坐标如表4.

在求得重力坝抗滑稳定极限状态方程式(36)在各 时段的设计验算点坐标后, 即可采用1.3节Pearson方法 计算各时段功能函数 $\{G(\tau, X(\tau, \omega)) \leq 0\}$ 与 $\{G(\tau+\Delta \tau, X(\tau$ $+\Delta \tau, \omega)) \leq 0\}$ 相关系数 $\rho_{G}(\tau, \tau+\Delta \tau)$, 其中 $\tau=10 \mathrm{a}$ 和 $\tau=70 \mathrm{a}$ 对应的极限状态方程相关系数如图 5 .

在求得不同时刻混凝土坝抗滑稳定可靠度指标以 及对应的极限状态方程相关系数后, 即可采用跨越率 计算公式(29)求解不同时刻极限状态方程跨越率, 进 而采用式(9)计算混凝土坝抗滑稳定累计失效概率, 相 关跨越率和累计失效概率计算结果如表 5 , 由表可知, 在上述老化失效条件假定下，随着服役时间的增长， 该混凝土坝累计抗滑稳定失效概率逐年增加, 运行70 年后, 累计失效概率达到 $3.28 \times 10^{-4}$.

值得注意的是, 本文在应用PHI2算法进行该混凝 土坝时变可靠度分析过程中, $\Delta \tau$ 取为 1 个月, 事实上, 在应用式(29)进行混凝土坝时变跨越率计算过程中, 时间增量步 $\Delta \tau$ 的选取对计算结果影响较大 ${ }^{[31,32]}$ : 当 $\Delta \tau$ 选取过大或过小时, 将会导致时变跨越率 $v^{+}(\tau)$ 计算收 玫过程不稳定, 进而导致计算精度降低. 基于此, 本文 结合式(29), 分别将时间增量步 $\Delta \tau$ 取为 $0.1,1,10,100$ 和 $1000 \mathrm{~d}$, 对 $t=0$ 初始时刻混凝土坝跨越率 $v^{+}(0)$ 进行计算, 相应 $v^{+}(t=0, \Delta \tau) / v^{+}(t=0,10)$ 随时间增量步 $\Delta \tau$ 演化曲线如 图6, 由图可知, 相比 $v^{+}(t=0, \Delta \tau) / v^{+}(t=0,10)=1$ 的理想情

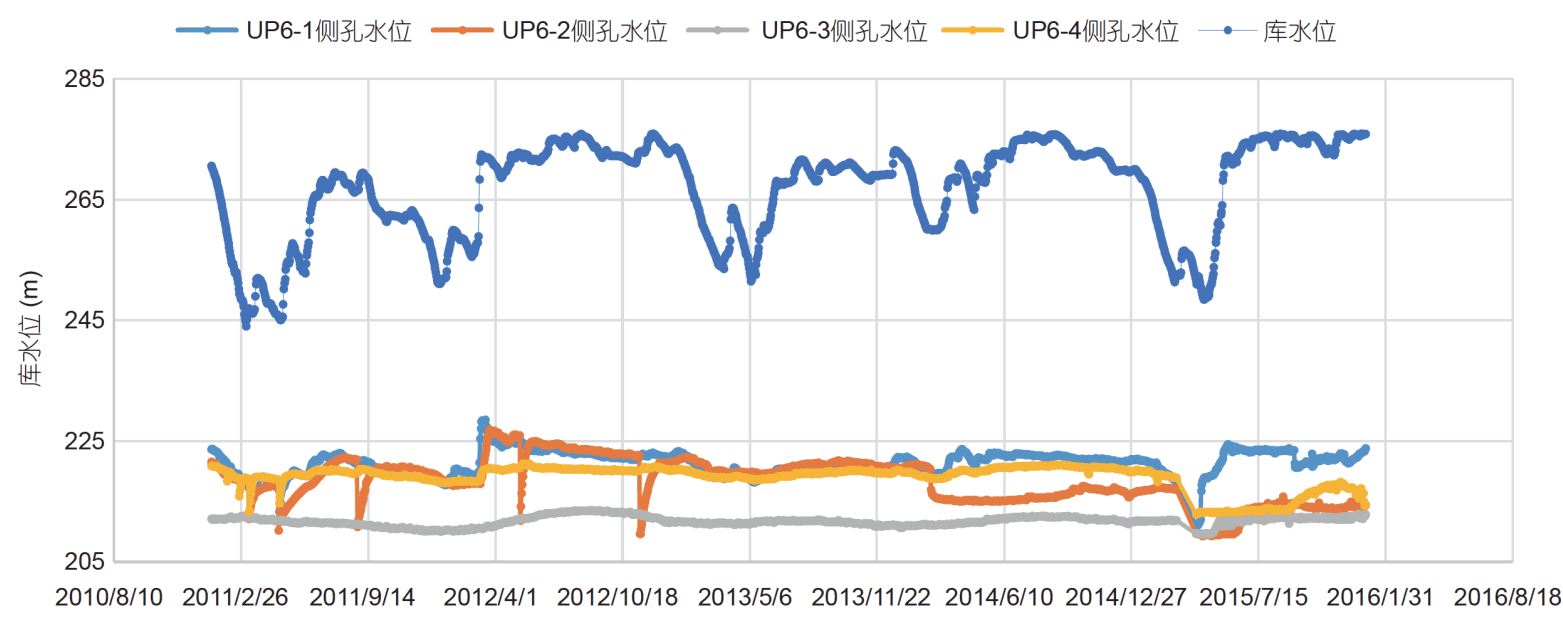

图 4 (网络版彩图)坝基扬压力测孔水位实测过程线 
表 1 上游历年年最高水位统计(单位: $\mathrm{m}$ )

\begin{tabular}{cc||cc}
\hline 年份 & 水位 & 年份 & 水位 \\
\hline 1995 & 273.46 & 2005 & 276.07 \\
1996 & 276.54 & 2006 & 275.27 \\
1997 & 272.23 & 2007 & 273 \\
1998 & 274.38 & 2008 & 275.99 \\
1999 & 275.38 & 2009 & 274.77 \\
2000 & 274.71 & 2010 & 276.01 \\
2001 & 275.94 & 2011 & 271.91 \\
2002 & 275.98 & 2012 & 275.95 \\
2003 & 271.93 & 2013 & 273.61 \\
2004 & 268.71 & 2014 & 275.84 \\
\hline
\end{tabular}

表 2 混凝土坝抗滑稳定主要随机变量及其统计特性

\begin{tabular}{cccc}
\hline 变量 & 分布类型 & 均值 & 标准差 \\
\hline 坝体自重 $\gamma_{c}\left(\mathrm{kN} / \mathrm{m}^{3}\right)$ & 正态 & 24.0 & 0.47 \\
坝/基初始摩擦系数 $f^{\prime}$ & 对数正态 & 1.35 & 0.14 \\
坝/基初始凝聚力 $c^{\prime}(\mathrm{MPa})$ & 对数正态 & 0.86 & 0.25 \\
下游水深 $H_{2}(\mathrm{~m})$ & 正态分布 & 13.4 & 0.43 \\
\hline
\end{tabular}

表 3 不同时段混凝土重力坝抗滑稳定可靠度指标

\begin{tabular}{cc}
\hline 时间 (a) & 可靠指标 $\beta$ \\
\hline 0 & 3.997 \\
5 & 3.912 \\
10 & 3.825 \\
20 & 3.791 \\
30 & 3.715 \\
40 & 3.601 \\
50 & 3.43 \\
60 & 3.366 \\
70 & 3.341 \\
\hline
\end{tabular}

表 4 验算点坐标

\begin{tabular}{cccccc}
\hline 时间 (a) & $H_{1}(\mathrm{~m})$ & $H_{2}(\mathrm{~m})$ & $\gamma_{\mathrm{c}}\left(\mathrm{kN} / \mathrm{m}^{3}\right)$ & $f^{\prime}$ & \multicolumn{1}{c}{$c^{\prime}(\mathrm{MPa})$} \\
\hline 0 & 74.376 & 13.245 & 23.928 & 1.327 & 0.846 \\
10 & 74.381 & 13.264 & 23.931 & 1.331 & 0.849 \\
20 & 74.415 & 13.328 & 23.934 & 1.342 & 0.851 \\
30 & 74.417 & 13.334 & 23.936 & 1.348 & 0.862 \\
40 & 74.436 & 13.345 & 23.941 & 1.352 & 0.874 \\
50 & 74.451 & 13.372 & 23.962 & 1.356 & 0.891 \\
60 & 74.582 & 13.418 & 23.975 & 1.371 & 0.893 \\
70 & 74.619 & 13.579 & 23.991 & 1.384 & 0.912 \\
\hline
\end{tabular}



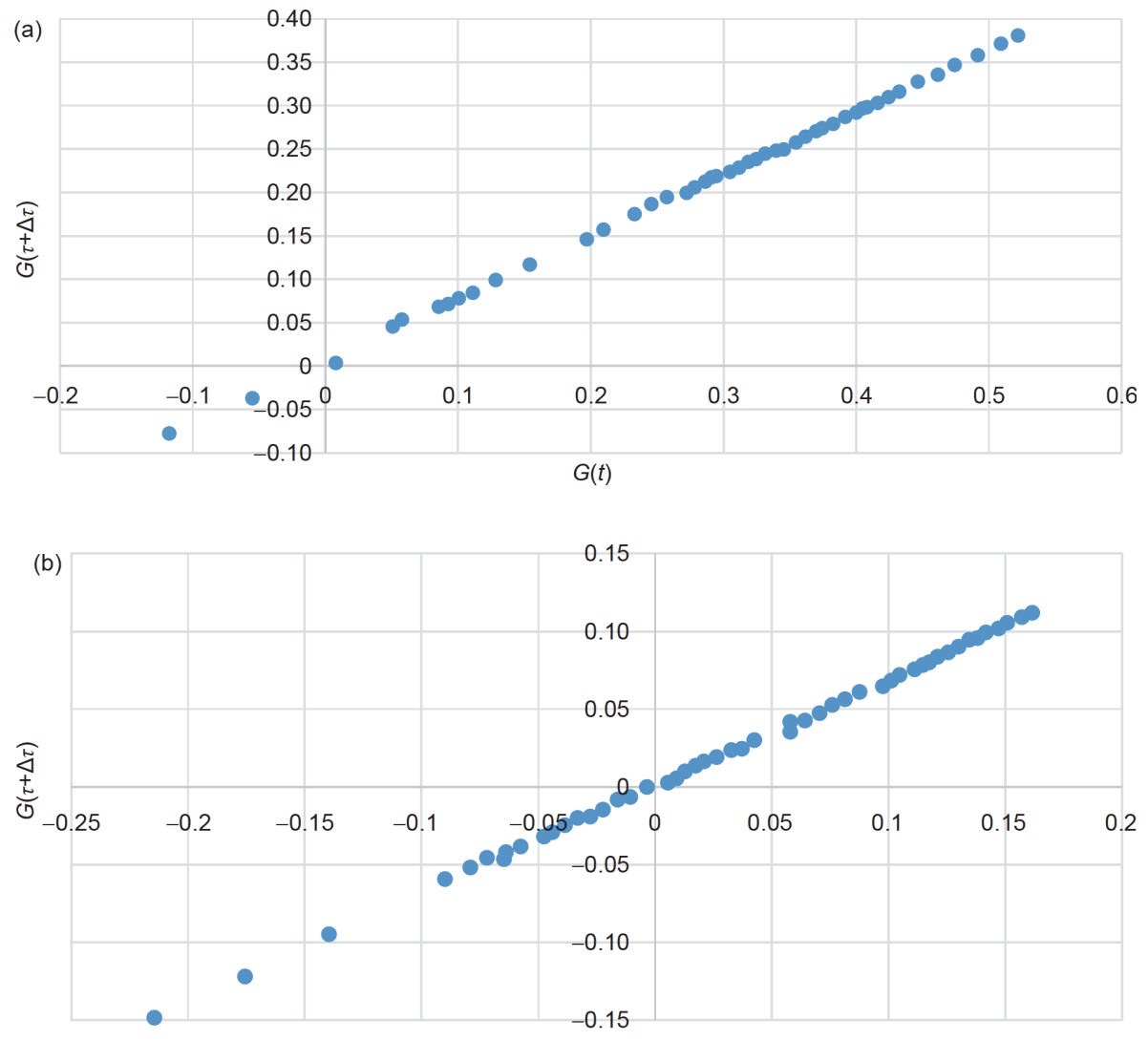

$G(t)$

图 5 (网络版彩图)不同服役时间下混凝土坝抗滑稳定极限状态方程相关系数. (a) $T=10$ a; (b) $T=70$ a

表 5 混凝土重力坝跨越率和累计失效概率计算结果统计

\begin{tabular}{ccc}
\hline 时间 (a) & 跨越率 & 累计失效概率 \\
\hline 0 & 0.00001 & 0.00001 \\
10 & 0.0000164 & 0.0000264 \\
20 & 0.0000265 & 0.0000434 \\
30 & 0.0000384 & 0.0000826 \\
40 & 0.0000575 & 0.000107 \\
50 & 0.0000784 & 0.000173 \\
60 & 0.0000879 & 0.000235 \\
70 & 0.0000956 & 0.000328 \\
\hline
\end{tabular}

况，当 $\Delta \tau<10 \mathrm{~d}$ 或者 $\Delta \tau>100 \mathrm{~d}$ 时，都将会导致计算得到 的混凝土坝失效跨越率 $v^{+}(\tau)$ 数值不稳定，而 $(10 \mathrm{~d}$, $100 \mathrm{~d})$ 相对为一个组较为合理的时间增量步取值范围, 因此, 本文算例选取 $\Delta \tau=30 \mathrm{~d}$ 可以认为在合理范围内.

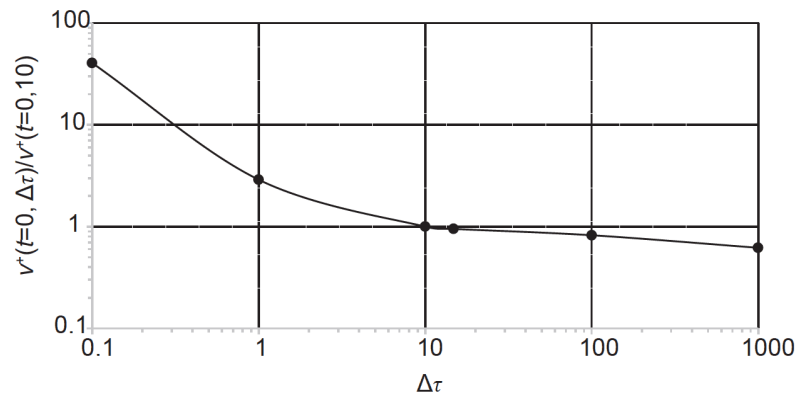

图 6 混凝土坝失效跨越率 $v^{+}(t)$ 相对时间增量步 $\Delta \tau$ 变化敏感 性分析. 横、纵坐标均为对数坐标

\section{5 结论}

混凝土坝通常分布于偏远的高山峡谷中，服役运 行环境恶劣，坝体无表面防护，而直接与水、大气和 岩体接触，坝身混凝土材料在长期承受各种静、动荷 载的同时，还需要长期遭受高压渗流、溶蚀、冲刷、 
冻融和热胀冷缩等有害作用，导致混凝土坝出现不同 程度的老化病变问题，造成工程病险频现、结构抗力 降低和时效风险率增大，若加之后期运行维护不当， 甚至可能导致出现大坝溃决等灾难性事故, 影响大坝 长效安全运行, 因此, 如何结合混凝土坝实际运行环 境和自身结构特点, 对混凝土坝时变风险率进行实时 在线评估成为了重要的研究课题.

本文针对混凝土坝时变风险率计算过程中的高维 非线性积分求解问题，尝试引入PHI2算法将混凝土坝 时变风险率分析问题转化为静态并联体系失效概率计
算问题，并通过在广义随机空间内采用JC法计算固定 时刻混凝土坝风险率，利用首次超越概率理论计算混 凝土坝失效事件跨越率和混凝土坝时变风险率. 在工 程算例应用中, 以某混凝土重力坝沿建基面抗滑失稳 失效为例, 通过引入合理的混凝土坝结构抗力和荷载 效应随机过程变量模型，采用上述算法计算出了混凝 土坝随着服役年限增加而逐渐增长时变跨越率和累计 失效概率. 该理论对于科学评估混凝土重力坝工作性 态, 并为实现大坝长效健康服役具有十分重要的理论 基础和工程应用价值.

\section{参考文献}

1 宋恩来. 混凝土坝老化与安全评价. 大坝与安全, 2008, 4: 4-8

2 吴中如. 中国大坝的安全和管理. 中国工程科学, 2000, 2: 36-39

3 顾冲时, 吴中如. 大坝与坝基安全监控理论和方法及其应用. 南京: 河海大学出版社, 2006

4 顾冲时, 苏怀智. 混凝土坝工程长效服役与风险评定研究述评. 水利水电科技进展, 2015, 35: 1-12

5 Vrijling J K. Probabilistic design of water defense systems in The Netherlands. Reliab Eng Syst Saf, 2001, 74: 337-344

6 Griffiths D V, Huang J, Fenton G A. Three dimensional probabilistic slope stability analysis by RFEM. In: Proceedings of the 17th International Conference on Soil Mechanics and Geotechnical Engineering: The Academia and Practice of Geotechnical Engineering. 2009

7 Kuo J T, Yen B C, Hsu Y C, et al. Risk analysis for dam overtopping-Feitsui reservoir as a case study. J Hydraul Eng, 2007, 133: 955-963

8 解家毕, 孙东亚. 事件树法原理及其在堤坝风险分析中的应用. 中国水利水电科学研究院学报, 2006, 4: 133-137

9 雷瑞丽, 张贵金, 陈雄波. 基于随机微分方程的大坝漫顶风险研究. 人民黄河, 2010, 32: 114-115, 117

10 彭慧慧. 重力坝坝基失稳模糊风险分析方法研究. 硕士学位论文. 大连: 大连理工大学, 2011

$11 \mathrm{Hu}$ J, Ma F H, Wu S H. Nonlinear finite-element-based structural system failure probability analysis methodology for gravity dams considering correlated failure modes. J Central South Univ, 2017, 24: 178-189

12 徐卫军, 侯建国, 李端有. 结构可靠度时变预测及灰色关联度在结构稳定分析中的应用. 长江科学院院报, 2005, 22: 20-23

13 姜树海, 范子武. 时变效应对大坝防洪风险率的影响研究. 水利学报, 2006, 37: 425-430

14 苏怀智, 顾冲时, 吴中如. 大坝工作性态的模糊可拓评估模型及应用. 岩土力学, 2006, 27: 2115-2121

15 杨跃新. 混凝土连续梁桥的时变可靠度评定与寿命预测. 硕士学位论文. 哈尔滨: 哈尔滨工业大学, 2007

, Tvedt L. Parallel system approach for vector out-crossing. J Offshore Mech Arct Eng, 1992, 114: 122-128

Hagen Ø, Tvedt L. Vector process out-crossing as parallel system sensitivity measure. J Eng Mech, 1991, 117: 2201-2220

Hagen Ø. Conditional and joint failure surface crossing of stochastic processes. J Eng Mech, 1992, 118: 1814-1839

Andrieu-Renaud C, Sudret B, Lemaire M. The PHI2 method: A way to compute time-variant reliability. Reliab Eng Syst Saf, 2004, 84: 75-86

$\mathrm{Hu}$ Z, Du X. Time-dependent reliability analysis with joint upcrossing rates. Struct Multidisc Optim, 2013, 48: 893-907

赵国藩, 王恒栋. 广义随机空间内的结构可靠度实用分析方法. 土木工程学报, 1996, 4: 47-51

李云贵, 赵国藩. 广义随机空间内的结构可靠度渐近分析方法. 水利学报, 1994, 8: 36-41

张俊芝. 服役工程结构可靠性理论及其应用. 北京: 中国水利水电出版社, 2007

Knodel P, Lo K, Ogawa T, et al. Measurements of strength parameters of concrete-rock contact at the dam-foundation interface. Geotech Test J, 1991, 14: 383

25 Electric Power Research Institute. Uplift pressures, shear strengths, and tensile strengths for stability analysis of concrete gravity dams. Technical Report. 1992

王宏硕, 陆述远, 廖孟扬. 混凝土与基岩胶结面的真实抗剪强度. 武汉大学学报(工学版), 1981, 4: 3-16

张俊芝, 李桂青. 服役重力坝系统可靠度及概率寿命探讨. 水利学报, 2000, 31: 40-45 
Pedro J O, Mascarenhas A, Silva H S. Ageing of concrete dam foundations. In: ISRM International Symposium-EUROCK. Lisboa, 1993. 401A

\title{
The research of the time-varying risk of concrete dam based on first transcendental probability theory
}

\author{
ZHU Kai ${ }^{1,2}$, ZHENG XueQin ${ }^{3}$, JI ZhuoHao ${ }^{1,2}$, FU ShuYan ${ }^{1,2,4}$, WU BangBin ${ }^{1,2}$ \& DAI Bo ${ }^{1,2}$ \\ ${ }^{1}$ State Key Laboratory of Hydrology-Water Resources and Hydraulic Engineering, Hohai University, Nanjing 210098, China; \\ ${ }^{2}$ National Engineering Research Center of Water Resources Efficient Utilization and Engineering Safety, Hohai University, Nanjing 210098, China; \\ ${ }^{3}$ Technology Center of State Grid Xinyuan Company Ltd., Beijing 100761, China; \\ ${ }^{4}$ College of Water Conservancy, Yunnan Agricultural University, Kunming 650201, China
}

With the gradual transition of dam safety management work from engineering safety management to engineering risk management in China, the dam risk degree has become a more and more important research field. Under the influence of mutiple random uncertain and fuzzy factors, the dam risk degree exhibits strong time-varying characteristics. This paper adopted the bivariate Gaussian method to compute the time-varying risk degree based on parallel-system method by transforming the time-varying risk computation into the risk computation of the failure of static parallel system. By computing the static dam risk degree at fixed times based on the JC method in generalized random space, the time-varying risk degrees were obtained based on first transcendental probability theory. Finally, the case study took a certain concrete gravity dam on the foundation interface as an example and the time-varying risk degree of the dam sliding failure was computed with the proposed method by introducing feasible random-process variable model of the resistance and load effects, verifying the effectiveness of the model, which is of certain theoretical and practical values.

concrete dam, time-varying risk degree, first transcendental probability, bivariate Gaussian method, randomprocess model

doi: 10.1360/N092018-00044 